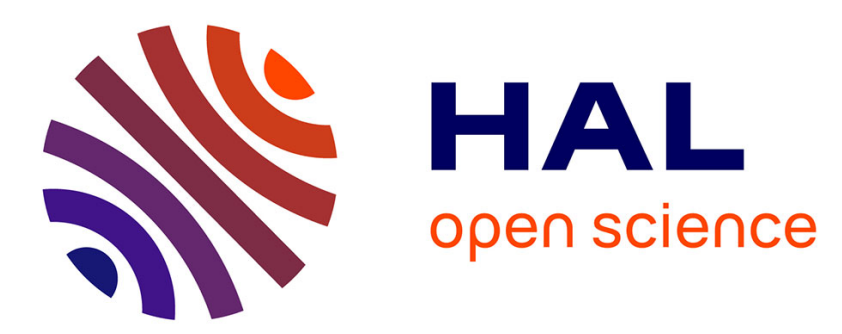

\title{
Normal form near orbit segments of convex Hamiltonian systems.
}

\author{
Shahriar Aslani, Patrick Bernard
}

\section{To cite this version:}

Shahriar Aslani, Patrick Bernard. Normal form near orbit segments of convex Hamiltonian systems.. International Mathematics Research Notices, 2022, 11, pp.8196-8208. 10.1093/imrn/rnaa344 . hal02550940v3

\section{HAL Id: hal-02550940 \\ https://hal.science/hal-02550940v3}

Submitted on 17 Jan 2022

HAL is a multi-disciplinary open access archive for the deposit and dissemination of scientific research documents, whether they are published or not. The documents may come from teaching and research institutions in France or abroad, or from public or private research centers.
L'archive ouverte pluridisciplinaire HAL, est destinée au dépôt et à la diffusion de documents scientifiques de niveau recherche, publiés ou non, émanant des établissements d'enseignement et de recherche français ou étrangers, des laboratoires publics ou privés. 


\section{Normal Form Near Orbit Segments of Convex Hamiltonian Systems}

\section{Shahriar Aslani and Patrick Bernard ,*}

PSL Research University, École Normale Supérieure, DMA (UMR CNRS 8553) 45, rue d'Ulm 75230 Paris Cedex 05, France

In the study of Hamiltonian systems on cotangent bundles, it is natural to perturb Hamiltonians by adding potentials (functions depending only on the base point). This led to the definition of Mañé genericity [8]: a property is generic if, given a Hamiltonian $H$, the set of potentials $g$ such that $H+g$ satisfies the property is generic. This notion is mostly used in the context of Hamiltonians that are convex in $p$, in the sense that $\partial_{p p}^{2} H$ is positive definite at each point. We will also restrict our study to this situation. There is a close relation between perturbations of Hamiltonians by a small additive potential and perturbations by a positive factor close to one. Indeed, the Hamiltonians $H+g$ and $H /(1-g)$ have the same level one energy surface, hence their dynamics on this energy surface are reparametrisation of each other, this is the Maupertuis principle. This remark is particularly relevant when $H$ is homogeneous in the fibers (which corresponds to Finsler metrics) or even fiberwise quadratic (which corresponds to Riemannian metrics). In these cases, perturbations by potentials of the Hamiltonian correspond, up to parametrisation, to conformal perturbations of the metric. One of the widely studied aspects is to understand to what extent the return map associated to a periodic orbit can be modified by a small perturbation. This kind of question depends strongly on the context in which they are posed. Some of the most studied contexts are, in increasing order of difficulty, perturbations of general vector fields, perturbations of Hamiltonian systems inside the class of Hamiltonian systems, perturbations of Riemannian metrics inside the class of Riemannian metrics, and Mañé perturbations 
of convex Hamiltonians. It is for example well known that each vector field can be perturbed to a vector field with only hyperbolic periodic orbits, this is part of the Kupka-Smale Theorem, see [5, 13] (the other part of the Kupka-Smale Theorem states that the stable and unstable manifolds intersect transversally; it has also been studied in the various settings mentioned above but will not be discussed here). In the context of Hamiltonian vector fields, the statement has to be weakened, but it remains true that each Hamiltonian can be perturbed to a Hamiltonian with only non-degenerate periodic orbits (including the iterated ones), see [11, 12]. The same result is true in the context of Riemannian metrics: every Riemannian metric can be perturbed to a Riemannian metric with only non-degenerate closed geodesics, this is the bumpy metric theorem, see $[1,2,4]$. The question was investigated only much more recently in the context of Mañé perturbations of convex Hamiltonians, see [9, 10]. It is proved in [10] that the same result holds: if $H$ is a convex Hamiltonian and $a$ is a regular value of $H$, then there exist arbitrarily small potentials $g$ such that all periodic orbits (including iterated ones) of $H+g$ at energy $a$ are non-degenerate. The proof given in [10] is actually rather similar to the ones given in papers on the perturbations of Riemannian metrics. In all these proofs, it is very useful to work in appropriate coordinates around an orbit segment. In the Riemannian case, one can use the so-called Fermi coordinates. In the Hamiltonian case, appropriate coordinates are considered in [10,Lemma 3.1] itself taken from [3, Lemma C.1]. However, as we shall detail below, the proof of this Lemma in [3], Appendix C, is incomplete, and the statement itself is actually wrong. Our goal in the present paper is to state and prove a corrected version of this normal form Lemma. Our proof is different from the one outlined in [3], Appendix C. In particular, it is purely Hamiltonian and does not rest on the results of [7] on Finsler metrics, as [3] did. Although our normal form is weaker than the one claimed in [10], it is actually sufficient to prove the main results of $[6,10]$, as we shall explain after the statement of Theorem 1, and probably also of the other works using [3, Lemma C.1].

\section{Introduction.}

When studying Mañé generic properties of convex Hamiltonians, a natural group of changes of coordinates to consider is the group of symplectic diffeomorphisms preserving the vertical fibration, that is, those symplectic diffeomorphisms of the form $\psi(q, p)=(\varphi(q), G(q, p))$. We call such transformations fibered. It is well known that such 
a diffeomorphism is symplectic if and only if the 2nd coordinate $G(q, p)$ is of the form

$$
G(q, p)=\alpha_{q}+p \circ\left(d \varphi_{q}\right)^{-1}
$$

for some closed one-form $\alpha$. We will say that the fibered symplectic diffeomorphism $\psi$ is homogeneous if it preserves the zero section, which is equivalent to the existence of a diffeomorphism $\varphi$ of the base such that

$$
\psi(q, p)=\left(\varphi(q), p \circ\left(d \varphi_{q}\right)^{-1}\right)
$$

We will say that the above diffeomorphism is vertical if it is of the form $(q, p) \longmapsto(q, p+$ $\alpha_{q}$ ), that is, if it preserves the 1st coordinate. Each fibered symplectic diffeomorphism is thus the composition of a vertical and of a homogeneous symplectic diffeomorphisms (in any order). Note that fibered symplectic diffeomorphisms preserve convexity, since their restrictions to fibers is affine.

If $\psi$ is a fibered symplectic diffeomorphism and $g(q)$ is a potential, then $(H \circ \psi)+$ $g=\left(H+g \circ \varphi^{-1}\right) \circ \psi$. So if a property is invariant under symplectic diffeomorphisms, for example having only non-degenerate periodic orbits on a given energy level, then this property is satisfied by $H+g$ for arbitrarily small $g$ if and only if it is satisfied by $g+H \circ \psi$ for arbitrarily small $g$.

These considerations allow us to enlarge the group of transformations considered in [3, Lemma C.1], where only homogeneous symplectic diffeomorphisms are considered. Although it is natural to restrict to homogeneous transformations in the case where $H$ is homogeneous, there is no reason to do so in general, and we will actually see that it is not possible to do so: allowing vertical symplectic transformations is necessary to obtain a nice normal form.

Since all considerations are local, we will always work on the manifold $\mathbb{R}^{1+d}$ and on its cotangent bundle $\mathbb{R}^{1+d} \times\left(\mathbb{R}^{1+d}\right)^{*}$. We will use the notations $q=\left(q_{0}, q_{*}\right) \in \mathbb{R} \times \mathbb{R}^{d}$ and similarly $p=\left(p_{0}, p_{*}\right) \in \mathbb{R}^{*} \times \mathbb{R}^{d *}$. We denote by $e_{0}, e_{1}, \ldots, e_{d}$ both the standard base of $\mathbb{R}^{1+d}$ and $\mathbb{R}^{(1+d) *}$.

If $(Q, P)$ is an orbit segment such that $\dot{Q}(0) \neq 0$, then there exists a local diffeomorphism $\varphi$ of the base that sends the orbit segment $Q(t), t \in[-\delta, \delta]$ to the straight line segment $t e_{0}, t \in[-\delta, \delta]$. Once this reduction has been performed, we only consider fiber-preserving symplectic diffeomorphisms $\psi$, which have the property that their horizontal component $\varphi$ is the identity on the segment $[-\delta, \delta] e_{0}$. We call such 
diffeomorphisms admissible. This implies that

$$
d \varphi_{t e_{0}} \cdot e_{0}=e_{0} \quad, \quad\left(p \circ\left(d \varphi_{t e_{0}}\right)^{-1}\right)_{0}=p_{0}
$$

where the 2nd equality is obtained by applying the linear form $p \circ d \varphi_{t e_{0}}^{-1}$ to the $1 \mathrm{st}$ equality, and where $p_{0}$ is the 1 st coordinate $p_{0}=p \cdot e_{0}$. In other words, the 1 st component $p_{0}$ of the momentum is not changed by applying an admissible homogeneous symplectic diffeomorphism.

Theorem 1. Let $\underline{H}: T^{*} \mathbb{R}^{1+d} \longrightarrow \mathbb{R}$ be a smooth Hamiltonian convex in $p$ in the sense that $\partial_{p p}^{2} \underline{H}$ is positive definite at each point. Let $(\underline{Q}(t), \underline{P}(t))$ be an orbit of $\underline{H}$ such that $\underline{\dot{Q}}(0) \neq 0$. Then there exists a smooth local fibered symplectic diffeomorphism $\psi$ and $\delta>0$ such that the new Hamiltonian $H=\underline{H} \circ \psi$ and the new orbit $(Q(t), P(t))=$ $\psi^{-1}(\underline{Q}(t), \underline{P}(t))$ satisfy, for all $t \in[-\delta, \delta]$,

$$
\begin{aligned}
Q(t) & =t e_{0} \\
P(t) & =0, \\
\partial_{p_{0} p_{*}}^{2} H\left(t e_{0}, P(t)\right) & =0, \\
\partial_{q p_{*}}^{2} H\left(t e_{0}, P(t)\right) & =0, \\
\partial_{p_{*} p_{*}}^{2} H\left(t e_{0}, P(t)\right) & =I d .
\end{aligned}
$$

If $\partial_{q_{*} p_{0}}^{2} H\left(t e_{0}, 0\right)$ does not identically vanish, then this quantity cannot be reduced to zero by applying an admissible change of coordinates preserving the other equalities.

Note that equality (3) can obviously not be obtained using only homogeneous changes of coordinates, since they preserve the zero section. Moreover, it follows from (1) that the 1 st coordinate $P_{0}(t)$ is invariant under the action of homogeneous admissible diffeomorphisms. As a consequence, it is not true that orbits of general convex Hamiltonians can be reduced to $(Q(t), P(t))=\left(t e_{0}, e_{0}\right)$ by such diffeomorphisms, as is claimed in [3, Lemma C.1]. This becomes possible (and easy) once vertical changes of coordinates are allowed, as we shall verify below.

Contrarily to [3, Lemma C.1], the last claim of the theorem implies that (5) can't be strengthened to $\partial_{q p}^{2} H\left(t e_{0}, 0\right)=0$, even if vertical symplectic diffeomorphisms, in 
addition to homogeneous ones, are permitted. See however Section 3 where this equality is proved in the homogeneous case.

If $H$ satisfies all the conclusions of Theorem 1, it is of the form

$$
H(q, p)=f(q)+w(q) p_{0}+\frac{1}{2} a\left(q_{0}\right) p_{0}^{2}+\frac{1}{2}\left\langle p_{*} p_{*}\right\rangle+O_{3}\left(q_{* \prime} p\right)
$$

where $f$ and $w$ are smooth functions from $\mathbb{R}^{1+d}$ to $\mathbb{R}$ satisfying $f\left(q_{0}, 0\right)=f(0,0)$ and $w\left(q_{0}, 0\right)=1$ for each $q_{0} \in[-\delta, \delta]$. Setting $\tilde{H}(q, p):=(H(q, p)-H(0)) / w(q)$, we thus have

$$
\tilde{H}(q, p)=\tilde{f}(q)+p_{0}+\frac{1}{2} \tilde{a}\left(q_{0}\right) p_{0}^{2}+\frac{1}{2}\left\langle p_{*^{\prime}} p_{*}\right\rangle+O_{3}\left(q_{* \prime} p\right),
$$

with $\tilde{f}\left(q_{0}, 0\right)=0$. This means that the additional conclusion $\partial_{q p}^{2} H\left(t e_{0}, 0\right)=0$ can be achieved provided we translate $H$ so that our orbit has energy 0 (which does not change anything to the dynamics), and then multiply $H$ by a function of $q$ (which is a reparametrisation of the dynamics on the energy surface $\left.\tilde{H}^{-1}(0)=H^{-1}(H(0))\right)$. This fact seems sufficient to derive most of the applications of [3,Lemma C.1] existing in the literature.

Let us illustrate for example how the results of [10] can be obtained. We denote by $E_{t}$ the space $\left\{q_{0}=t\right\} \cap\left\{p_{0}=0\right\}$, which projects isomorphically to $\mathbb{R}^{d} \times \mathbb{R}^{d *}$. Let $L: E_{0} \longrightarrow E_{\delta}$ be the differential at zero of the transition maps between the sections $\left\{q_{0}=0\right\} \cap\{\tilde{H}=0\}$ and $\left\{q_{0}=\delta\right\} \cap\{\tilde{H}=0\}$, seen as a symplectic $2 d \times 2 d$ matrix. Since the dynamics of $H$ and $\tilde{H}$ in restriction to $\tilde{H}^{-1}(0)$ are reparametrisations of each other, they have the same transition map hence $L_{H}=L_{\tilde{H}}$. The main statement of [10] is that, if $X$ is a dense set of symplectic $2 d \times 2 d$ matrices, there exist arbitrarily small potentials $g(q)$ such that $L_{H+g} \in X$. This statement is proved in [10] for Hamiltonian having the form (8) above, and the normal form is invoked, to reduce each Hamiltonian to this form. However, as we have explained, one can only obtain the normal form (7) in general. The missing step is to deduce the statement for $H$ from the statement for $\tilde{H}$, which turns out to be easy: applying the statement to $\tilde{H}$ gives small potentials $\tilde{g}(q)$ such that $L_{\tilde{H}+\tilde{g}} \in X$. We now observe that $\tilde{H}+\tilde{g}=(H-H(0)+w \tilde{g}) / w$, hence

$$
L_{H+w \tilde{g}}=L_{\tilde{H}+\tilde{g}} \in X
$$

Since the function $w$ depends only on $q, w \tilde{g}$ is a potential, which can be made arbitrarily small by taking $\tilde{g}$ arbitrarily small. We have proved the statement for $H$. 


\section{Proof of the Normal Form.}

We will always work in coordinates such that (2) holds and consider only admissible changes of coordinates (i.e., changes of coordinates whose horizontal component fixes the axis $\left.\mathbb{R} e_{0}\right)$. Our proof is purely Hamiltonian and does not rest on [7, Lemma 3.1]. Actually, a small modification of the proof also implies this Lemma, as will be explained in the next section. We will apply several admissible diffeomorphisms. At each step, we will denote by $\underline{H}$ the initial Hamiltonian and by $H=\underline{H} \circ \psi$ the transformed Hamiltonian. In the matrix computations below, we most of the time consider the momenta $p$ as line matrices hence denote by $p M$ what might also be denoted by $M^{t} p$.

Proof of (3). Let $\underline{P}_{0}(t)$ be the 1st component of $\underline{P}(t)$ (the orbit before the change of coordinates). We consider a function $v(t): \mathbb{R} \longrightarrow \mathbb{R}$ such that $v^{\prime}=\underline{P}_{0}$ and the function $u\left(q_{0}, q_{*}\right):=v\left(q_{0}\right)+\underline{P}_{*}\left(q_{0}\right) \cdot q_{*}$. We have $d u_{t e_{0}}=\underline{P}(t)$, hence applying the vertical diffeomorphism $\psi(q, p)=\left(q, p+d u_{q}\right)$, the new orbit $(Q(t), P(t))=\psi^{-1}(\underline{Q}(t), \underline{P}(t))$ satisfies $P(t)=0$.

Proof of (4). We assume that (3) and (2) are already satisfied for $\underline{H}$ and prove that (4) can be obtained by a further change of coordinates. We consider a base diffeomorphism of the form $\varphi\left(q_{0}, q_{*}\right)=\left(q_{0}+l\left(q_{0}\right) \cdot q_{*}, q_{*}\right)$, where $q_{0} \longmapsto l\left(q_{0}\right)$ is a smooth map with values in $\mathbb{R}^{d *}$. The corresponding homogeneous diffeomorphism satisfies

$$
\psi:\left(q_{0}, 0, p_{0}, p_{*}\right) \longmapsto\left(q_{0}, 0, p_{0}, p_{*}-p_{0} l\left(q_{0}\right)\right)
$$

We then have $\partial_{p_{*}}(\underline{H} \circ \psi)_{\left(q_{0} e_{0}, p_{0}, p_{*}\right)}=\partial_{p_{*}} \underline{H}_{\left(q_{0} e_{0}, p_{0}, p_{*}-p_{0} l\left(q_{0}\right)\right)}$ hence

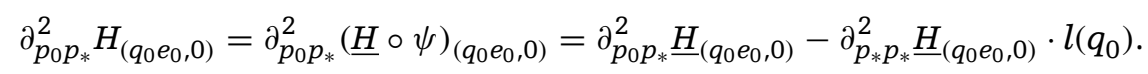

We obtain (4) by choosing

$$
l\left(q_{0}\right):=\left(\partial_{p_{*} p_{*}}^{2} \underline{H}_{\left(q_{0} e_{0}, 0\right)}\right)^{-1} \cdot \partial_{p_{0} p_{*}}^{2} \underline{H}_{\left(q_{0} e_{0}, 0\right)} \cdot
$$

Observe that $\partial_{p_{*} p_{*}}^{2} H_{\left(q_{0} e_{0}, 0\right)}$ is invertible because $\partial_{p p}^{2} H$ is positive definite at each point.

Proof of (5). This equality can be obtained by a further homogeneous change of coordinates preserving (2) and (4). We could assume (3), but, keeping in mind another 
application in Section 3, we only make the slightly more general assumption that $\underline{P}(t) \equiv \underline{P}(0)=\left(P_{0}, 0\right)$ for some constant $P_{0}$. We consider the vector field

$$
\underline{V}(q):=\partial_{p} \underline{H}(q, P(0))
$$

on $\mathbb{R}^{d+1}$. We apply a variant of the Flow Box Theorem to the vector field $\underline{V}$. More precisely, we consider the diffeomorphism $\varphi(q)=\left(q_{0}, \phi\left(q_{0}, q_{*}\right)\right)$, defined in a neighborhood of $[-\delta, \delta] \times\{0\}$ in such a way that $q_{*} \longmapsto \phi\left(t, q_{*}\right)$ is the transition map along the orbits of $\underline{V}$ between the sections $\left\{q_{0}=0\right\}$ and $\left\{q_{0}=t\right\}$. In other words, $\varphi\left(q_{0}, q_{*}\right)=\Phi^{q_{0}}\left(0, q_{*}\right)$, where $\Phi^{t}$ is the flow of the reparametrised vector field $\underline{V}(q) / \underline{V}_{0}(q)\left(\underline{V}_{0}\right.$ is the 1st coordinate of $\underline{V})$. It is a smooth diffeomorphism near $[-\delta, \delta] \times\{0\}$, and $\underline{V}=\underline{V}_{0} \varphi_{\sharp} e_{0}$, where $\varphi_{\sharp} e_{0}$ is the forward image of the constant vector field $e_{0}$.

Since $\varphi$ is fixing the axis $\mathbb{R} e_{0}$ and preserving $q_{0}$, the associated homogeneous diffeomorphism $\psi$ preserves (2) and (4). Moreover, $\psi(q, P(0))=(\varphi(q), P(0))$. Denoting as usual $H:=\underline{H} \circ \psi$, and $V(q)=\partial_{p} H(q, P(0))$, we have $\varphi_{\sharp} V=\underline{V}=\underline{V}_{0} \varphi_{\sharp} e_{0}$, hence $V=\left(\underline{V}_{0} \circ \varphi^{-1}\right) e_{0}$, and $V_{*}=0$, or in other words $\partial_{p_{*}} H(q, P(0))=0$ for all $q$. Differentiating with respect to $q$ gives (5).

Proof of (6). We assume that the equations (3) to (5) initially hold. We will obtain (6) by an admissible (usually not homogeneous) transformation preserving all these equalities. This transformation will be decomposed into first a homogeneous transformation and second a vertical transformation none of which preserve (5).

The 1st step consists of applying the homogeneous change of coordinates $\psi$ associated to a diffeomorphism of the form

$$
\varphi\left(q_{0}, q_{*}\right)=\left(q_{0}, M\left(q_{0}\right) \cdot q_{*}\right)
$$

where $M(t)$ is a $d \times d$ invertible matrix depending smoothly on $t$. The matrix of the differential of $\varphi$ is

$$
D(q)=\left[\begin{array}{cc}
1 & 0 \\
M^{\prime}\left(q_{0}\right) q_{*} & M\left(q_{0}\right)
\end{array}\right], \quad D^{-1}(q)=\left[\begin{array}{cc}
1 & 0 \\
-M^{-1}\left(q_{0}\right) M^{\prime}\left(q_{0}\right) q_{*} & M^{-1}\left(q_{0}\right)
\end{array}\right]
$$

where $M^{\prime}\left(q_{0}\right)$ is the derivative. We thus have

$$
\psi(q, p)=\left(q_{0}, M\left(q_{0}\right) q_{*}, p_{0}-p_{*} M^{-1}\left(q_{0}\right) M^{\prime}\left(q_{0}\right) q_{*}, p_{*} M^{-1}\left(q_{0}\right)\right)
$$


The Hamiltonian in original coordinates is of the form

$$
\underline{H}(q, p)=\underline{H}(q, 0)+\underline{v}(q) p_{0}+\frac{1}{2} \underline{a}\left(q_{0}\right) p_{0}^{2}+\frac{1}{2}\left\langle p_{*} \underline{A}\left(q_{0}\right), p_{*}\right\rangle+O_{3}\left(q_{*}, p\right),
$$

with $\underline{v}(q)=\partial_{p_{0}} \underline{H}(q, 0), \underline{a}\left(q_{0}\right)=\partial_{p_{0} p_{0}}^{2} \underline{H}\left(q_{0} e_{0}, 0\right), \underline{A}\left(q_{0}\right)=\partial_{p_{*} p_{*}}^{2} \underline{H}\left(q_{0} e_{0}, 0\right)$. We compute

$$
\begin{aligned}
H(q, p)=\underline{H} \circ \psi(q, p) & =H(q, 0)+v(q) p_{0}-p_{*} M^{-1}\left(q_{0}\right) M^{\prime}\left(q_{0}\right) q_{*}+\frac{1}{2} a\left(q_{0}\right) p_{0}^{2} \\
& +\frac{1}{2}\left\langle p_{*} M^{-1}\left(q_{0}\right) \underline{A}\left(q_{0}\right), p_{*} M^{-1}\left(q_{0}\right)\right\rangle+O_{3}\left(q_{*} p\right) .
\end{aligned}
$$

We get (6) provided $M\left(q_{0}\right) M^{t}\left(q_{0}\right)=\underline{A}\left(q_{0}\right)$ for each $q_{0}$. We could for example take $M\left(q_{0}\right)=\underline{A}^{1 / 2}\left(q_{0}\right)$ for each $q_{0}$ (remember that $\underline{A}\left(q_{0}\right)$ is positive definite for each $\left.q_{0}\right)$. However, the unavoidable apparition of the term $p_{*} M^{-1}\left(q_{0}\right) M^{\prime}\left(q_{0}\right) q_{*}$ means that (5) have been destroyed. In order to be able to restore it by a vertical change of coordinates, we need a better choice for $M\left(q_{0}\right)$ :

Lemma 2.1. We can choose $M\left(q_{0}\right)$ in such a way that

$$
H(q, p)=H(q, 0)+v(q) p_{0}-p_{*} B\left(q_{0}\right) q_{*}+\frac{1}{2} a\left(q_{0}\right) p_{0}^{2}+\frac{1}{2}\left\langle p_{* \prime} p_{*}\right\rangle+O_{3}\left(q_{* \prime} p\right),
$$

where $B\left(q_{0}\right)$ is symmetric for each $q_{0}$.

Proof. We need the matrix $M(t)$ to satisfy the two conditions that $M(t) M^{t}(t)=\underline{A}(t)$ and $B(t):=M^{-1}(t) M^{\prime}(t)$ is symmetric. Derivating the 1 st condition, we get $M^{\prime} M^{t}+M\left(M^{\prime}\right)^{t}=\underline{A^{\prime}}$. Using the symmetry of $B$, we deduce that $\left(M^{t}\right)^{\prime}\left(M^{t}\right)^{-1}=M^{-1} M^{\prime}$, hence that $M\left(M^{t}\right)^{\prime}=$ $M M^{-1} M^{\prime} M^{t}=M^{\prime} M^{t}$. We obtain the equation $2 M^{\prime} M^{t}=\underline{A^{\prime}}$ or in other words:

$$
M^{\prime}(t)=\underline{A}^{\prime}(t)\left(M^{t}\right)^{-1} / 2
$$

Reducing $\delta$ if necessary, there exists a solution $M(t)$ of this equation on the interval $[-\delta, \delta]$, satisfying moreover the initial condition $M(0)=\underline{A}^{1 / 2}(0)$. For such a solution, we see that

$$
M^{-1} M^{\prime}=M^{-1} \underline{A^{\prime}}\left(M^{t}\right)^{-1} / 2=\left(M^{\prime}\right)^{t}\left(M^{t}\right)^{-1}
$$


hence the corresponding matrix $B$ is indeed symmetric. Then, the same computation made earlier shows that $\left(M M^{t}\right)^{\prime}=\underline{A}^{\prime}$. Since the equality $M M^{t}=\underline{A}$ is satisfied at $t=0$, it is thus satisfied for all $t \in[-\delta, \delta]$.

The 2nd step consists of applying the vertical change of coordinates

$$
\Psi:(q, p) \longmapsto\left(q, p+d u_{q}\right)
$$

with $u(q)=\left\langle B\left(q_{0}\right) q_{*}, q_{*}\right\rangle / 2$, so that $d u_{q}=\left(*, B\left(q_{0}\right) q_{*}\right)$. It is then a direct computation that

$$
H \circ \Psi(q, p)=f(q)+w(q) p_{0}+\frac{1}{2} a\left(q_{0}\right) p_{0}^{2}+\frac{1}{2}\left\langle p_{* \prime} p_{*}\right\rangle+O_{3}\left(q_{* \prime} p\right)
$$

for some smooth functions $f$ such that $f\left(q_{0}, 0\right)=H(0,0)$, and $w$ such that $w\left(q_{0}, 0\right)=1$.

We now prove the last statement of the theorem about the impossibility of achieving the additional condition $\partial_{q_{*} p_{0}}^{2} H=0$. We shall only consider admissible diffeomorphisms that preserve (2), (3), and (4). Every fibration preserving symplectic diffeomorphism preserving (2) and (3) is the composition of a homogeneous and a vertical diffeomorphisms each of which preserve (2) and (3).

Let us first observe that $\partial_{q_{*} p_{0}}^{2} H\left(t e_{0}, 0\right)$ can't be changed by applying a vertical diffeomorphism preserving (3). Such a diffeomorphism is of the form $\psi(q, p)=(q, p+$ $d u_{q}$ ) for some smooth function $u$ satisfying $d u_{t e_{0}}=0$, hence in particular $u$ is constant on $\mathbb{R} e_{0}$. Then,

$$
\partial_{p_{0}}(H \circ \psi)(q, p)=\partial_{p_{0}} H\left(q, p+\partial_{q} u(q)\right)
$$

and

$$
\begin{aligned}
\partial_{q_{*} p_{0}}^{2}(H \circ \psi)\left(t e_{0}, 0\right) & =\partial_{q_{*} p_{0}}^{2} H\left(\left(t e_{0}, 0\right)+\sum_{i=0}^{d} \partial_{p_{i} p_{0}}^{2} H\left(t e_{0}, 0\right) \partial_{q_{*} q_{i}}^{2} u\left(t e_{0}\right)\right. \\
& =\partial_{q_{*} p_{0}}^{2} H\left(\left(t e_{0}, 0\right)+\partial_{p_{0} p_{0}}^{2} H\left(t e_{0}, 0\right) \partial_{q_{*} q_{0}}^{2} u\left(t e_{0}\right)=\partial_{q_{*} p_{0}}^{2} H\left(t e_{0}, 0\right)\right.
\end{aligned}
$$

In this computation, we have used first that $\partial_{p_{*} p_{0}}^{2} H\left(t e_{0}, 0\right)=0$, and then that $\partial_{q_{*} q_{0}}^{2} u\left(t e_{0}\right)=\partial_{q_{0} q_{*}}^{2} u\left(t e_{0}\right)=0$, which holds since $\partial_{q_{*}} u\left(t e_{0}\right)$ is identically zero.

We now consider the action of homogeneous admissible transformations. 
Lemma 2.2. The homogeneous symplectic diffeomorphism $\psi$ associated to $\varphi$ preserves (2) and (4) if and only if the matrix of the differential of $\varphi$ has the following $1+d$ block form for each $t \in[-\delta, \delta]$ :

$$
D \varphi\left(t e_{0}, P(t)\right)=\left[\begin{array}{ll}
1 & 0 \\
0 & *
\end{array}\right]
$$

Proof. Since $\varphi$ is admissible, the matrix $D \varphi$ along the orbit has the triangular block form

$$
D(t):=D \varphi\left(t e_{0}, P(t)\right)=\left[\begin{array}{ll}
1 & b(t) \\
0 & B(t)
\end{array}\right], \quad D^{-1}(t)=\left[\begin{array}{cc}
1 & -b(t) B^{-1}(t) \\
0 & B^{-1}(t)
\end{array}\right],
$$

hence

$$
\left.\psi(q, p)=\left(\varphi(q), p_{0}, p_{*} B^{-1}(t)-p_{0} b(t) B^{-1}(t)\right)\right)
$$

We have

$$
\partial_{p p}^{2}(H \circ \psi)\left(t e_{0}, P(t)\right)=D^{-1}(t) \partial_{p p}^{2} H_{\psi\left(t e_{0}, P(t)\right)}\left(D^{-1}\right)^{t}(t)
$$

In matrix form,

$$
\begin{aligned}
\partial_{p p}^{2}(H \circ \psi) & =\left[\begin{array}{cc}
1 & -b(t) B^{-1}(t) \\
0 & B^{-1}(t)
\end{array}\right]\left[\begin{array}{cc}
\partial_{p_{0} p_{0}}^{2} H & 0 \\
0 & \partial_{p_{*} p_{*}}^{2} H
\end{array}\right]\left[\begin{array}{cc}
1 & 0 \\
-\left(B^{-1}\right)^{t}(t) b^{t}(t) & \left(B^{-1}\right)^{t}(t)
\end{array}\right] \\
& =\left[\begin{array}{cc}
* & -b(t) B^{-1}(t) A(t)\left(B^{-1}\right)^{t} \\
B^{-1}(t) A(t)\left(B^{-1}\right)^{t} b^{t} & B^{-1}(t) A(t)\left(B^{-1}\right)^{t}
\end{array}\right] .
\end{aligned}
$$

This matrix is block diagonal (which is equivalent to $\partial_{p_{0} p_{*}}^{2}(H \circ \psi)=0$ ) if and only if $b(t)=0$.

Let now $\underline{H}$ be a Hamiltonian satisfying (2) to (4), and $\psi$ be the homogeneous transformation associated to the diffeomorphism $\varphi$. We assume that $H=\underline{H} \circ \psi$ still satisfies (2) to (4). We denote by $a(q)$ the 1 st coordinate of $\varphi$. In view of the previous Lemma, we have the block diagonal form for $t \in[-\delta, \delta]$,

$$
D \varphi_{\left(t e_{0}, 0\right)}=\left[\begin{array}{cc}
1 & 0 \\
0 & Z(t)
\end{array}\right]
$$


in particular $\partial_{q} a\left(q_{0}, 0\right)=e_{0}, \partial_{q_{*}} a\left(q_{0}, 0\right)=0$ for each $q_{0} \in[-\delta, \delta]$ thus $\partial_{q_{0} q_{*}}^{2} a\left(q_{0}, 0\right)=0$. It is convenient for the following computations to denote $V(q):=\partial_{p} H(q, 0), \underline{V}(q):=\partial_{p} \underline{H}(q, 0)$, so that

$$
\underline{V}(\varphi(q))=d \varphi_{q} \cdot V(q)
$$

and, focusing on the 1st coordinates, $\underline{V}_{0}(\varphi(q))=\partial_{q} a(q) \cdot V(q)$. Differentiating with respect to $q_{*}$ at the point $t e_{0}, t \in[-\delta, \delta]$ yields

$$
\begin{aligned}
\partial_{q_{*}} \underline{V}_{0}\left(t e_{0}\right) \cdot Z(t) & =\partial_{q_{*} q}^{2} a\left(t e_{0}\right) \cdot V\left(t e_{0}\right)+\partial_{q} a\left(t e_{0}\right) \cdot \partial_{q_{*}} V\left(t e_{0}\right) \\
& =\partial_{q_{*}}^{2} a\left(t e_{0}\right) \cdot e_{0}+\partial_{q_{*}} V_{0}\left(t e_{0}\right)=\partial_{q_{*} q_{0}}^{2} a\left(t e_{0}\right)+\partial_{q_{*}} V_{0}\left(t e_{0}\right) \\
& =\partial_{q_{*}} V_{0}\left(t e_{0}\right) .
\end{aligned}
$$

Since $Z(t)$ is invertible for each $t \in[-\delta, \delta]$, we have proved that $\partial_{q_{*} p_{0}}^{2} H\left(t e_{0}, 0\right)=$ $\partial_{q_{*}} V_{0}\left(t e_{0}\right)=0$ if and only if $\partial_{q_{*} p_{0}}^{2} \underline{H}\left(t e_{0}, 0\right)=\partial_{q_{*}} \underline{V}_{0}\left(t e_{0}\right)=0$. If $\underline{H}$ did not satisfy this condition from the start, then neither does $H$.

\section{The Homogeneous Case}

We explain here for completeness how the arguments given above also imply the following statement, which is equivalent to [7, Lemma 3.1].

Proposition 3.1. Let $\underline{H}: T^{*} \mathbb{R}^{1+d} \longrightarrow \mathbb{R}$ be a Hamiltonian positively homogeneous in the fibers, smooth and positive outside of the zero section, and such that $\partial_{p p}\left(H^{2}\right)$ is positive definite at each point outside of the zero section. Let $(\underline{Q}(t), \underline{P}(t))$ be an orbit of $\underline{H}$ such that $\underline{\dot{Q}}(0) \neq 0$. Then there exists a smooth local homogeneous symplectic diffeomorphism $\psi$ and $\delta>0$ such that the new Hamiltonian $H=\underline{H} \circ \psi$ and the new orbit $(Q(t), P(t))=\psi^{-1}(\underline{Q}(t), \underline{P}(t))$ satisfy the equalities (2), (4), and (5) for each $t \in[-\delta, \delta]$, as well as

$$
\begin{aligned}
P(t) & =\left(P_{0}(0), 0\right), \\
\partial_{q p}^{2} H\left(t e_{0}, P(t)\right) & =0 .
\end{aligned}
$$

Proof. We work in coordinates where (2) hold and apply admissible homogeneous transformations. 
The 1st component $P_{0}(t)$ of the momentum is independent of $t$, and non-zero. Indeed, we have, using the Euler relation,

$$
P_{0}(t)=P(t) \cdot e_{0}=P(t) \cdot \partial_{p} H\left(t e_{0}, P(t)\right)=a H\left(t e_{0}, P(t)\right),
$$

where $a$ is the degree of homogeneity. Then,

$$
P_{0}(t)=a H\left(t e_{0}, P(t)\right)=a H(0, P(0))=P_{0}(0),
$$

where we have used the preservation of $H$ along the orbit. Moreover, the constant $P_{0}(t)$ as well as the energy $H\left(t e_{0}, P(t)\right)$ are non-zero otherwise the orbit would be constant.

We now apply the same homogeneous diffeomorphism as in the proof of (4) above, with $l\left(q_{0}\right)=\underline{P}_{*}(t) / P_{0}$, where $P_{0}=\underline{P}_{0}$ is the 1 st component of the momentum. In view of (9), we get (10).

Then (4) automatically holds: we have

$$
\partial_{p_{*}} H\left(t e_{0},\left(P_{0}, 0\right)\right)=\partial_{p_{*}} H\left(t e_{0}, P(t)\right)=\dot{Q}_{*}(t)=0 .
$$

By homogeneity of the map $p \longmapsto \partial_{p_{*}} H\left(t e_{0}, p\right)$, we deduce that $\partial_{p_{*}} H\left(t e_{0},\left(s P_{0}, 0\right)\right)=0$ for each $s>0$, hence that $\partial_{p_{0} p_{*}}^{2} H\left(t e_{0},\left(p_{0}, 0\right)\right)=0$.

The equality (5) can then be obtained by applying a homogeneous change of coordinates, the proof of the previous section can directly be applied.

The equality $\partial_{q p_{0}} H\left(t e_{0}, P(t)\right)=0$, hence (11), then automatically holds. Indeed, since $P(t)=P(0)$ is constant, we have $\partial_{q} H\left(t e_{0},\left(P_{0}(t), 0\right)\right)=\partial_{q} H\left(t e_{0}, P(t)\right)=\dot{P}(t)=0$. Using the homogeneity of the map $p \longmapsto \partial_{q} H\left(t e_{0}, p\right)$, we deduce that $\partial_{q} H\left(t e_{0},\left(s P_{0}, 0\right)\right)=0$ for each $s>0$, hence that $\partial_{p_{0} q} H\left(t e_{0},\left(P_{0}, 0\right)\right)=0$.

\section{Funding}

This work was supported by the European Union's Horizon 2020 research and innovation programme under the Marie Skłodowska-Curie grant agreement [754362].

\section{References}

[1] Abraham, R. "Bumpy metrics, global analysis (Berkeley, Calif., 1968)." Proc. Sympos. Pure Math. 14 (1970): 1-3.

[2] Anosov, D. V. "Generic properties of closed geodesics (Russian)." Izv. Akad. Nauk SSSR Ser. Mat. 46 (1982): 675-709. 896; translation: Mathematics of the USSR-Izvestiya, 21 (1983), No. 1. 
[3] Figalli, A. and L. Rifford. “Closing Aubry sets II." Comm. Pure Appl. Math. 68 (2015): 345-412.

[4] Klingenberg, W. and F. Takens. "Generic properties of geodesic flows." Math. Ann. 197 (1972): 323-34.

[5] Kupka, I. "Contribution à la théorie des champs génériques. (French)." Contrib. Diff. Equ. 2 (1963): 457-84.

[6] Lazrag, A., L. Rifford, and R. Ruggiero. "Franks' lemma for C2-Mañé perturbations of Riemannian metrics and applications to persistence." J. Mod. Dyn. 10 (2016): 379-411.

[7] Li, Y. and L. Nirenberg. "The distance function to the boundary, Finsler geometry, and the singular set of viscosity solutions of some Hamilton-Jacobi equations." Comm. Pure Appl. Math. 58 (2005): 85-146.

[8] Mañé, R. "Generic properties and problems of minimizing measures of Lagrangian systems." Nonlinearity 9 (1996): 273-310.

[9] Oliveira, E. "Generic properties of Lagrangians on surfaces: the Kupka-Smale theorem." DCDS 21 (2008): 551-69.

[10] Rifford, L. and R. Ruggiero. "Generic properties of closed orbits of Hamiltonian flows from Mañés viewpoint." Int. Math. Res. Not. 22 (2012): 5246-65.

[11] Robinson, C. "Generic properties of conservative systems I." Am. J. Math. 92 (1970): 562-603.

[12] Robinson, C. "Generic properties of conservative systems I." Am. J. Math. 92 (1970): 897-906.

[13] Smale, S. "Stable manifolds for differential equations and diffeomorphisms." Ann. Scuola Norm. Sup. Pisa Cl. Sci. 17 (1963): 97-116. 